\title{
Core collapse supernovae and starbursts
}

\author{
Miguel A. Pérez-Torres* \\ Instituto de Astrofísica de Andalucía - CSIC, 18080 Granada, Spain \\ E-mail: torres@iaa.es
}

Core-collapse supernovae (CCSNe; Type Ib/c and Type II SNe) are the endproducts of massive stars $\left(\mathrm{M} \geq 8 \mathrm{M}_{\odot}\right)$, and yield radio events whose brightness depends on the intensity of the interaction experienced by the supernova ejecta with the circumstellar presupernova wind material [1]. The fact that CCSNe are intrinsically radio supernovae -albeit with a huge range of different radio powers- and hence unaffected by dust absorption, together with the high resolution and high sensitivity provided by current VLBI arrays, has been exploited to directly image the radio brightness structure of CCSNe in nearby $(D \lesssim 20 \mathrm{Mpc})$ galaxies. This has allowed to gain insight into the physics of both CCSNe and of the circumstellar medium (CSM) with which they interact. In addition, ultra-high-resolution, ultra-high-sensitivity radio observations of CCSNe in Luminous and Ultra-Luminous Infrared Galaxies (LIRGs and ULIRGs, respectively) in the local Universe, can be used to directly measure of the current CCSN rate and star formation rate (SFR), given an assumed initial mass function (IMF).

In this contribution, I give a brief overview of VLBI observations made of some CCSNe in nearby galaxies, and then present some of the most relevant results obtained with high-resolution radio observations of (U)LIRGs in the local Universe, aimed at directly detecting CCSNe via their radio emission, and thus determine their CCSN and star formation rates, independently of models. This is of particular relevance, in view of the fact that our estimates of star formation (and CCSN) rates in high-z starburst galaxies relies on standard relationships between far-infrared luminosity and star-formation rate. In particular, I will present recently obtained results with the e-EVN on the nuclear region of Arp 299-A.

Science and Technology of Long Baseline Real-Time Interferometry:

The 8th International e-VLBI Workshop, EXPReSO9

June 22 - 262009

Madrid, Spain

${ }^{*}$ Speaker. 


\section{Circumstellar interaction and VLBI imaging of core-collapse supernovae in nearby galaxies}

Type II supernovae ( $\mathrm{SNe}$ ) are associated with massive stars that have expelled slow, dense winds during their supergiant phase. The stellar explosion drives a shock into this wind, at speeds as high as $20000 \mathrm{~km} \mathrm{~s}^{-1}$ and temperatures of $\sim 10^{9} \mathrm{~K}$. In addition, a reverse shock propagates back into the stellar envelope at speeds of $500-1000 \mathrm{~km} \mathrm{~s}^{-1}$ relative to the expanding ejecta. This is the so-called standard interaction model [1], and radio, optical, and X-ray emission from Type II supernovae have been usually interpreted within this model. The outgoing shock forms a highenergy-density shell that is responsible for the production of synchrotron radio emission, while the reverse shock accounts for the optical and soft X-ray emission.

A beautiful confirmation that the above scenario applies to Type II SNe came when SN 1993J (Type IIb) in M81 (D $\approx 3.63 \mathrm{Mpc}$ ) was imaged with VLBI just six months after its explosion, showing a shell-like radio structure [2]. Its first year of expansion proceeded in a self-similar way [3], in perfect agreement with predictions from the standard interaction model. The continuous monitoring of SN 1993J with VLBI arrays at centimeter wavelengths have allowed to characterize its expansion and determine the properties of the shock/CSM interaction [ [ 5].

No less spectacular than SN 1993J is the case of SN 1987A in the Large Magellanic Cloud ( $D \approx 150 \mathrm{kpc}$ ). SN 1987A faded away very quickly (a few days) at radio wavelengths, indicating that the shock front had gone past the presupernova wind, which was a weird behaviour for a Red Super Giant (RSG) progenitor. When it was clear that SN 1987A went off while in its BSG phase, it was predicted that once the shock front would reach the RSG wind of the progenitor star, we would then witness a rise in flux density. In fact, SN 1987A reemerged at radio wavalengths in mid 1990, and images taken in 1996 and later on, showed a remarkably circularly symmetric structure, as displayed by SN 1993J. Recently, SN 1987A was successfully imaged with e-VLBI (Tingay et al., see these proceedings).

VLBI observations of SN 1986J (Type IIn) in NGC $891(D \approx 9.6 \mathrm{Mpc})$ taken approximately 16 yr after its explosion showed that SN 1986J has a distorted, rather than circularly symmetric, shell of radio emission, indicative of a deformation of the shock front, and probably due to the collision of the supernova ejecta with an anisotropic, clumpy medium [6]. Further multi-wavelength VLBI observations of SN 1986J have shown that this supernova hosts a compact object in its center [7], tentatively identified with a pulsar, the remnant left after the explosion of the progenitor star. SN 1979C (Type II L) in M100 ( $D \approx 16.1 \mathrm{Mpc})$ is another remarkable supernova that in the last years has shown an increasing flux density. This has allowed for VLBI imaging of its fine radio structure and for a monitoring that has permitted to determine its deceleration parameter, $m(r \propto$ $t^{m}$ ). SN 2001gd (Type IIb) and SN 2004et (Type IIP) have also been imaged with VLBI, but the large distance and relatively quick evolution of SN 2001gd [8] and the very faint event yielded by SN 2004et [9] resulted in VLBI images which do not resolve adequately their radio brightness structure.

\section{High-resolution radio imaging of starburst galaxies}

A large fraction of the massive star-formation at both low- and high- $z$ has taken place in 
(U)LIRGs. Thus, their implied high star-formation rates (SFRs) are expected to result in CCSN rates a couple of orders of magnitude higher than in normal galaxies. Therefore, a powerful tracer for starburst activity in (U)LIRGs is the detection of CCSNe, since the SFR is directly related to the CCSN rate. However, most SNe occurring in ULIRGs are optically obscured by large amounts of dust in the nuclear starburst environment, and have therefore remained undiscovered by (optical) SN searches. Fortunately, it is possible to discover these CCSNe through high-resolution radio observations, as radio emission is free from extinction effects. Furthermore, CCSNe are expected, as opposed to thermonuclear SNe, to become strong radio emitters when the $\mathrm{SN}$ ejecta interact with the circumstellar medium (CSM) that was ejected by the progenitor star before its explosion as a supernova. Therefore, if (U)LIRGs are starburst-dominated, bright radio $\mathrm{SNe}$ are expected to occur and, given its compactness and characteristical radio behaviour, can be pinpointed with high-resolution, high-sensitivity radio observations (e.g., SN 2000ft in NGC 7469 [10]; SN 2004ip in IRAS 18293-3413, [11]; SN 2008cs in IRAS 17138-1017, [12], [13]; supernovae in Arp 299 [14], Arp 220 [15, 16, 17], Mrk 273 [18]). However, since (U)LIRGs are likely to have an AGN contribution, it is mandatory the use of high-sensitivity, high-resolution radio observations to disentangle the nuclear and stellar (mainly from young $\mathrm{SNe}$ ) contributions to the radio emission, thus probing the mechanisms responsible for the heating of the dust in their (circum-)nuclear regions.

One of the most remarkable cases among (U)LIRGs is the prototypical ULIRG Arp 220. Its central region is composed of two radio nuclei, each of them hosting a large number of compact components, mostly identified with young SNe and SNRs [15, 16, 17]. The radio light curves of many of those $\mathrm{SNe}$ appeared to be stable over periods $\geq 5 \mathrm{yr}$, indicating their interaction with a dense ISM [19]. The eastern nucleus hosts $20 \mathrm{RSNe}$, while the western one hosts $29 \mathrm{RSNe}$, and its about three times brighter, suggesting that there might be a differential free-free absorption in the nuclei [16]. Recently, [17] have detected many of the compact components in Arp 220 at frequencies above $1.6 \mathrm{GHz}$, and their spectra indicate the presence of both relatively young $\mathrm{SNe}$ along with SNRs. Moreover, the radio supernova rate found by [17] coincides with the expected CCSN rate (4 SN/yr) as inferred from Arp 220 infrared luminosity. Since all the new RSNe are very bright, indicating they are Type IIn-like $\mathrm{SNe}$ (whose progenitor stars are very massive), this indicate a top-heavy IMF for the stars in the nuclei of Arp 220.

\section{3. e-VLBI imaging of Arp 299-A}

Arp 299 (the merging system formed by IC 694 and NGC 3690) is the "original" starburst galaxy (Gehrz et al. 1983) and an obvious merger system that has been studied extensively at many wavelengths. An active starburst in Arp 299 is indicated by the high frequency of recent optically discovered supernovae in the outer regions of the galaxy. Since the far infrared luminosity of Arp 299 is $L_{I R} \approx 6.5 \times 10^{11} L_{\odot}$, the implied CCSN rate is of $\approx 1.7 \mathrm{SN} / \mathrm{yr}$. Given that $50 \%$ of its total infrared emission comes from source A (see Fig. 1), it is expected that roughly $1 \mathrm{SN} / \mathrm{yr}$ will explode in region A. Therefore, this region is the one that shows most promises for finding new supernovae. Indeed, Neff et al. (2004) found a new component in this region, by comparing VLBA observations carried out in April 2002 and February 2003.

We proposed e-EVN observations aimed at detecting the radio emission from recently exploded SNe in Arp 299. Our observations, carried out in April 2008 and December 2008 at 5.0 
$\mathrm{GHz}$, resulted in the deepest images ever of Arp 299-A (see Figure 1). We found 26 compact components above $5 \sigma \mathrm{rms}$ noise, whose nature can be only explained if they are $\mathrm{SNe}$ and/or SNRs, likely embedded in super star clusters, and may challenge the standard scenario that directly links far-infrared luminosity to a CCSN and star formation rate, since the apparent rate of CCSNe might be much higher than expected. We leave, however, a detail discussion of this and other issues for future publications.

\section{Summary and discussion}

VLBI observations of nearby CCSNe have allowed for a better understanding of the physics, namely the determination of the deceleration parameter, interaction between ejecta and presupernova wind, characterization of the mass loss history and -sometimes- the explosion scenario, estimation of magnetic field and energy budget in fields and particles. However, this wealth of information has been obtained only for those supernovae that are bright $\left(L_{\text {peak }} \gtrsim 1.5 \times 10^{27} \mathrm{erg} \mathrm{s}^{-1} \mathrm{~Hz}^{-1}\right)$, long-lasting (radio lifetimes of a few years at least) and close enough $(D<20 \mathrm{Mpc})$, so that VLBI observations can adequately resolve and monitor their expansion. If we consider that normal galaxies have small CCSN rates ( $\lesssim 0.01 \mathrm{SN} / \mathrm{yr}$ ), and that most CCSNe (Type IIP and Type IIb) have radio peaks of a few times $10^{26} \mathrm{erg} \mathrm{s}^{-1} \mathrm{~Hz}^{-1}$ at most, this explains why so few radio supernovae have been observed with VLBI in the last $20 \mathrm{yrs}$, despite the VLBI arrays increasing their sensitivity. One importan way in which e-VLBI may contribute significantly in this field is in the prompt response that it offers. For example, Type Ib/c SNe, recently linked to long GRBs, are known to be rapidly evolving ( $t_{\text {peak }} \approx 10-20$ days) radio supernovae. Currently VLBI arrays do not offer the needed dynamic scheduling to, e.g., react on a nearby event, while e-VLBI offers such flexibility and, thanks to its ability to carry out real-time correlation, allows for a potential follow-up of the most interesting targets.

The CCSN rate in (U)LIRGs is expected to be at least one or two orders of magnitude larger than in normal galaxies [20], and hence detections of SNe in (U)LIRGs offer a promising way of determining the current star formation rate in nearby galaxies. However, the direct detection of CCSNe in the extreme ambient densities of the central few hundred pc of (U)LIRGs is extremely difficult, as the optical and IR emission of supernovae is severely hampered by the huge amounts of dust present in those regions, and can at best yield an upper limit to the true CCSN rate. Fortunately, it is possible to directly probe the star forming activity in the innermost regions of (U)LIRGs by means of high angular resolution, high-sensitivity radio searches of CCSNe, as radio does not suffer from dust obscuration. Current VLBI (and e-VLBI) arrays are starting to yield astonishing results, thanks to their few- $\mu \mathrm{Jy}$ sensitivity and milliarcsec resolution at $\mathrm{cm}$-wavelengths. In particular, the findings in Arp 220 using global VLBI and Arp 299 (using the e-EVN), may challenge the standard scenario that directly links far-infrared luminosity to a CCSN and star formation rate, which is of much relevance for studies of starburst galaxies at high-z.

Acknowledgments The author acknowledges support by the Spanish Ministerio de Educación y Ciencia (MEC) through grant AYA 2006-14986-C02-01, and also by the Consejería de Innovación, Ciencia y Empresa of Junta de Andalucía through grants FQM-1747 and TIC-126. MAPT is a Ramón y Cajal Post Doctoral Research Fellow funded by the MEC and the Consejo Superior de Investigaciones Científicas 

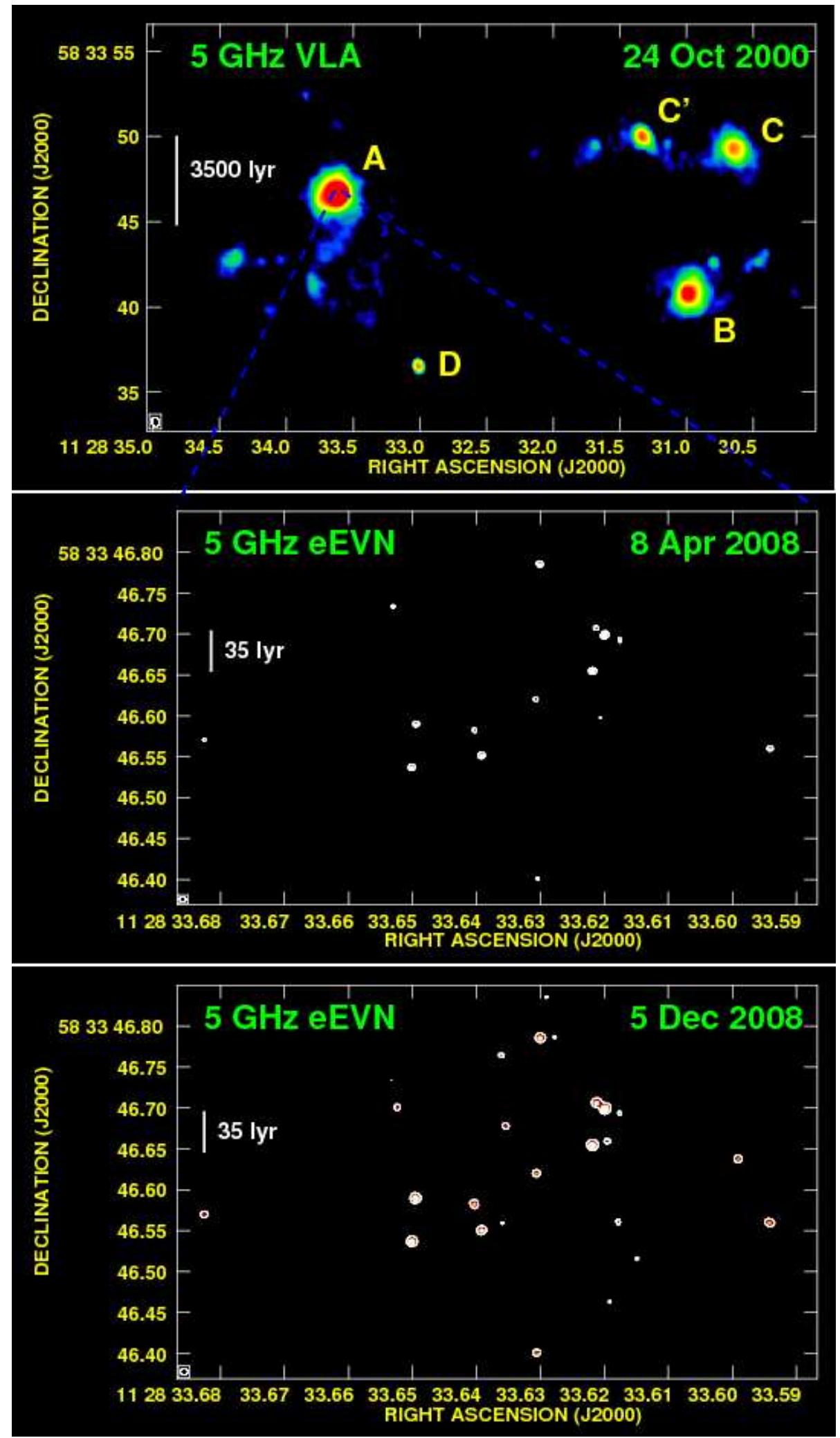

Figure 1: Top panel: $5 \mathrm{GHz}$ VLA archival observations of Arp 299 on 24 October 2000, displaying the five brightest knots of radio emission in this merging galaxy. Middle and bottom panels: $5 \mathrm{GHz}$ e-EVN observations of the central 500 light years of Arp 299-A on 8 April 2008 and 5 December 2008. The offsource root-mean-square (r.m.s.) noise level is $39 \mu \mathrm{Jy} / \mathrm{beam}$ and $25 \mu \mathrm{Jy} / \mathrm{beam}$ for the middle and bottom panels, respectively, and show the existence of 15 and 26 compact components with a signal-to-noise ratio (s.n.r.) equal or larger than five on 8 April 2008 and 5 December 2008, respectively. The size of the FWHM synthesized interferometric beam was of $(0.6 \operatorname{arcsec} \times 0.4 \mathrm{arcsec})$ for the VLA observations, and of (7.3 milliarcsec $\times 6.3$ milliarcsec) and ( 8.6 milliarcsec $\times 8.4$ milliarcsec) for the e-EVN observations on 8 April 2008 and 5 December 2008, respectively. 
(CSIC). The European VLBI Network is a joint facility of European, Chinese, South African and other radio astronomy institutes funded by their national research councils. e-VLBI developments in Europe are supported by the EC DG-INFSO funded Communication Network Developments project 'EXPReS', Contract No. 02662.

\section{References}

[1] R. Chevalier 1982, The Radio and X-ray Emission from Type II Supernovae, ApJ, 259, 302

[2] J.M. Marcaide et al. 1995a, Discovery of shell-like radio-structure in SN 1993J, Nature, 373, 44

[3] J.M. Marcaide et al. 1995b, Expansion of SN 1993J, Science, 270, 1475

[4] J.M. Marcaide et al. 1997, Deceleration in the expansion of SN 1993J, ApJ, 486, L31

[5] N. Bartel et al. 2000, The changing morphology and increasing deceleration of SN 1993J in M81, Science, 287, 112

[6] M.A. Pérez-Torres et al. 2002, A distorted radio shell in the young supernova SN 1986J, MNRAS, 335, L23

[7] M. Bietenholz et al. 2004, Discovery of a Radio Compact Component in the Center of SN 1986J, Science, 304, 1947

[8] M.A. Pérez-Torres et al. 2005, High-resolution observations of SN 2001gd in NGC 5033, MNRAS, 360,1055

[9] I. Marti-Vidal et al. 2007, 8.4 GHz VLBI observations of SN 2004et in NGC 6946, A\&A, 470, 1071

[10] L. Colina et al. 2001, Discovery of a Bright Radio Supernova in the Circumnuclear Starburst of the Luminous Infrared Seyfert 1 Galaxy NGC 7469, ApJ, 553, L19

[11] M.A. Pérez-Torres et al. 2007, Radio Detection of Supernova 2004ip in the Circumnuclear Region of the Luminous Infrared Galaxy IRAS 18293-3413, ApJ, 671, L21

[12] M.A. Pérez-Torres et al. 2008, Supernova 2008cs in IRAS 17138-1017, CBET, 1392, 2P

[13] E. Kankare et al. 2008, Discovery of a very highly extinguished supernova in a luminous infrared galaxy,

[14] Neff et al. 2004, A Supernova Factory in the Merger System Arp 299, ApJ, 611, 186

[15] H.E. Smith et al. 1998, A Starburst Revealed-Luminous Radio Supernovae in The Nuclei of Arp 220,ApJ, 493, L17

[16] Lonsdale et al. 2006, VLBI Images of 49 Radio Supernovae in Arp 220ApJ, 647, L185

[17] Parra et al. 2007, The Radio Spectra of the Compact Sources in Arp 220: A Mixed Population of Supernovae and Supernova Remnants, ApJ, 659, 314

[18] M. Bondi et al. 2005, A supernova factory in Mrk 273?, MNRAS, 361, 748

[19] E. Rovilos et al. 2005, The 18-cm light curves of the luminous radio supernova candidates in Arp 220,MNRAS, 359, 827

[20] Condon, J.J. 1992, Radio Emission from Normal Galaxies, ARAA, 30, 575

ApJ, 689, L97 